\title{
Intestinal permeability in relation to birth weight and gestational and postnatal age
}

\author{
R M van Elburg, W P F Fetter, C M Bunkers, H S A Heymans
}

Arch Dis Child Fetal Neonatal Ed 2003;88:F52-F55

See end of article for authors' affiliations

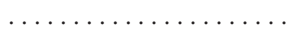

Correspondence to: Dr van Elburg, Department of Neonatology, VU University Medical Center, PO Box 7057, 1007 MB Amsterdam, The Netherlands; rm.vanelburg@vumc.nl

Accepted 30 April 2002

\begin{abstract}
Objective: To determine the relation between intestinal permeability and birth weight, gestational age, postnatal age, and perinatal risk factors in neonates.

Study design: Intestinal permeability was measured by the sugar absorption test within two days of birth and three to six days later in preterm and healthy term infants. In the sugar absorption test, the urinary lactulose/mannitol ratio is measured after oral ingestion of a solution (375 mosm) of lactulose and mannitol.

Results: A first sugar absorption test was performed in 116 preterm (26-36 weeks gestation) and 16 term infants. A second test was performed in 102 preterm and nine term infants. In the preterm infants, the lactulose/mannitol ratio was not related to gestational age $(r=-0.09, p=0.32)$ or birth weight $(r=0.07, p=0.43)$. The median lactulose/mannitol ratio was higher if measured less than two days after birth than when measured three to six days later $(0.427$ and 0.182 respectively, $p<0.001)$. The lactulose/mannitol ratio was higher in preterm infants than term infants if measured within the first 2 days of life $(0.404$ and 0.170 respectively, $p<0.001)$, but not different three to six days later $(0.182$ and 0.123 respectively, $p=0.08$ ). In multiple regression analysis of perinatal risk factors, only umbilical arterial $\mathrm{pH}$ correlated with the lactulose/mannitol ratio in preterm infants less than 2 days of age $(T=-1.98, p=0.05)$.

Conclusions: In preterm infants (26-36 weeks gestation), intestinal permeability is not related to gestational age or birth weight but is higher during the first 2 days of life than three to six days later. It is higher in preterm infants than in healthy term infants only if measured within two days of birth. This suggests rapid postnatal adaptation of the small intestine in preterm infants.
\end{abstract}

M aturation of the gastrointestinal tract is a dynamic process that is still in progress at birth. The small intestine serves as a selective barrier, which is reflected by selective permeability to macromolecules. As the barrier function is still developing at birth, increased intestinal permeability is a normal finding in the neonatal period. ${ }^{1-3}$ Increased intestinal permeability can have beneficial effects, such as the uptake of larger (nutritional) molecules and the development of systemic tolerance, but also disadvantageous effects, such as increased uptake of infectious agents leading to the development of infection, inflammation, and systemic hypersensitivity. ${ }^{4}$ Several factors, including gestational age, postnatal age, and the kind of enteral nutrition, have been suggested to play a role in the maturation process of the small intestine, resulting in a decrease in intestinal permeability. ${ }^{5}$

So far, only a few studies have measured intestinal permeability with a sugar absorption test (SAT) in term ${ }^{267}$ and preterm $^{189}$ infants. In only one study were both preterm and term infants investigated. ${ }^{3}$ In all studies except one ${ }^{9}$, a steady state method was used. We have previously shown that a SAT, using a single load hyperosmolar solution (1560 mosm) with lactulose and mannitol, in both healthy and ill children and adults is a simple, non-invasive test of intestinal permeability with good reproducibility. ${ }^{10-14}$ In the SAT, urinary excretion of a larger molecule, lactulose, and smaller molecule, mannitol, is measured after oral ingestion. In healthy control subjects, the lactulose/mannitol $(\mathrm{L} / \mathrm{M})$ ratio is low $(<0.09)$ because permeability to the larger molecule lactulose is much lower than permeability to the smaller molecule mannitol. ${ }^{11}{ }^{14}$ Under pathological conditions, the L/M ratio can be increased as the result of increased permeability to lactulose and/or decreased permeability to mannitol. As the use of hyperosmolar solutions in preterm infants is thought to be associated with necrotising enterocolitis, ${ }^{15}$ we modified the test solution for the use in neonates.

The aim of our study in preterm and term infants was to determine: $(a)$ if intestinal permeability is related to gestational age and birth weight; $(b)$ if intestinal permeability is related to postnatal age by measuring intestinal permeability within two days of birth and three to six days later; $(c)$ which perinatal factors influence intestinal permeability measured less than two days after birth in preterm infants.

\section{PATIENTS AND METHODS \\ Patients}

During the period November 1995 to December 1996, all preterm infants with a gestational age $<37$ weeks admitted to the neonatal intensive care unit were eligible for the study. Infants with complete absence of urine production before the test, suspicion of necrotising enterocolitis or perforation of the bowel, or major congenital abnormalities such as congenital heart defects, bowel atresia, and omphalocele were excluded. All infants were fed according to the feeding protocol of the neonatal ward, varying from daily $6 \times 1 \mathrm{ml}$ mother's milk or preterm formula in infants with acute or chronic hypoxia to daily $8 \times 10 \mathrm{ml}$ in near term healthy infants on the first day after birth.

As controls, we used term infants delivered by elective caesarean section after 37 weeks of gestational age. We chose these infants because they stay on the maternity ward during the recovery of their mothers, which usually takes about five

Abbreviations: SAT, sugar absorption test; L/M, lactulose/mannitol; NTISS, neonatal therapeutic intervention scoring system 


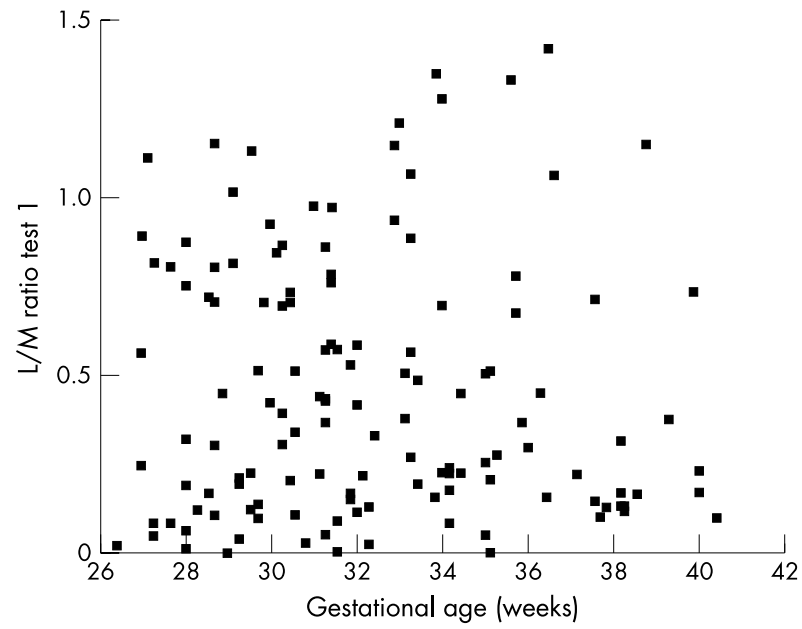

Figure 1 Lactulose/mannitol (L/M) ratio in 116 preterm infants, measured within two days of birth, plotted against gestational age. They are not related $(r=-0.09, p=0.32)$.

days. Therefore, these infants are available for testing twice. All term infants had normal Apgar scores ( 10 after 5 min) and all did well in the neonatal period.

\section{Methods}

The SAT was performed as previously described, ${ }^{11}$ with slight modifications related to the study population. The test solution was prepared under sterile conditions. Instead of a hyperosmolar solution, a less hyperosmolar (375 mosm) solution was used by omitting sucrose from the test solution. The test solution contained $250 \mathrm{mg}$ lactulose and $100 \mathrm{mg}$ mannitol per $5 \mathrm{ml}$ water. Fasting before the test was not mandatory. The test solution was instilled by nasogastric tube $(2 \mathrm{ml} / \mathrm{kg}$ body weight). Urine was then collected for six hours. Chlorhexidine digluconate $(0.1 \mathrm{ml}$ of a $20 \%$ solution) was added to the urine as a preservative. The urine volume was measured and samples were stored at $-20^{\circ} \mathrm{C}$. The concentrations of lactulose and mannitol were measured (in $\mathrm{mmol} / \mathrm{mol}$ creatinine) in the samples by gas chromatography as previously described, ${ }^{16}$ and the $\mathrm{L} / \mathrm{M}$ ratio was calculated. Reference values for the $\mathrm{L} / \mathrm{M}$ ratio with the hyperosmolar test solution in both older infants and adults are $0-0.089 .{ }^{11}$ In infants, complete collection of urine may be difficult. In our study, complete urine collection was achieved in $36 \%$ of the infants. Incomplete urine collection did not seem to influence the results of the study, therefore we combined the results for infants with and without complete urine collection as long as at least $5 \mathrm{ml}$, the minimum volume required for analysis, was collected.

\section{Study protocol}

After informed parental consent, infants were tested within two days of birth. A second test was performed three to six days after the first test. Perinatal factors, 5 min Apgar score, umbilical arterial $\mathrm{pH}$ (if available), birth weight, gestational age, enteral feeding in $\mathrm{ml} / \mathrm{kg} /$ day, and the neonatal therapeutic intervention scoring system (NTISS) ${ }^{17}$ were recorded. The study protocol was approved by the hospital medical ethics committee of the Isala Clinics, Location Sophia, Zwolle.

\section{Statistical analysis}

Data were analysed with SPSS 9.0 (SPSS Inc, Chicago, Illinois, USA). They are given as median values and range unless indicated otherwise. The difference between the median L/M ratio on the first test and the second test was tested with the nonparametric Wilcoxon signed ranks test for paired data. Correlations were determined using Pearson's correlation coefficient. To evaluate the influence of perinatal factors on

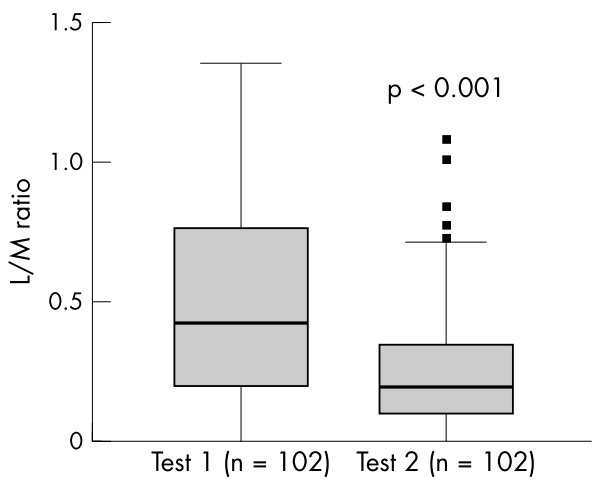

Figure 2 Lactulose/mannitol (L/M) ratio in 102 preterm infants measured within two days of birth (test 1) and three to six days later (test 2). It was higher in test $1(0.427)$ than in test $2(0.182)$ $(p<0.001)$.

intestinal permeability, multiple regression analysis was performed. A two sided $p$ value $<0.05$ was considered significant.

\section{RESULTS}

\section{Preterm infants}

In 116 preterm infants, 68 boys and 48 girls, the SAT was performed within two days of birth, at a median postnatal age of 28.5 hours (range 4-48). The median gestational age was 31 weeks and 2 days (range 26 weeks and 3 days to 36 weeks and 4 days), and birth weight was 1425 g (range 665-2720). In $102 / 116(88 \%)$ preterm infants, a second SAT was performed at a postnatal age of 141 hours (range 91-180). In 14 infants, the second SAT could not be performed for reasons such as early transfer to another hospital, failure to collect urine, or death of the infant. Urine was collected for all six hours in $41 / 116(35 \%)$ preterm infants in the first test and 37/102 $(36 \%)$ preterm infants in the second test.

The $\mathrm{L} / \mathrm{M}$ ratio of the first SAT was not related to gestational age $(r=-0.09, \mathrm{p}=0.32$; fig 1$)$, birth weight $(r=0.07$, $\mathrm{p}=0.43$ ), or postnatal age (hours) at which the first test was performed $(r=-0.13, \mathrm{p}=0.15)$. If the preterm infants are divided into two groups $(<34$ weeks and $>34$ weeks gestation), no differences between the two groups were found in the $\mathrm{L} / \mathrm{M}$ ratio measured within two days of birth or three to six days later.

The $\mathrm{L} / \mathrm{M}$ ratio of the first SAT was higher than that of the second SAT (0.427 and 0.182 respectively, p < 0.001; fig 2). The decrease in $\mathrm{L} / \mathrm{M}$ ratio was due to both increased permeability to mannitol $(\mathrm{p}<0.002)$ and, to a lesser extent, decreased permeability to lactulose $(\mathrm{p}<0.02)$. The $\mathrm{L} / \mathrm{M}$ ratio of the second SAT was not related to the postnatal age at which the second SAT was performed $(r=-0.025, \mathrm{p}=0.80)$.

If "critically ill" preterm infants, defined as infants on a ventilator, suffering from asphyxia, and/or born small for gestational age, were compared with "healthy" preterm infants, no differences in intestinal permeability were found either at the first test or the second test. The NTISS did not correlate with the $\mathrm{L} / \mathrm{M}$ ratio at either the first SAT $(r=-0.13$, $\mathrm{p}=0.17)$ or the second $(r=-0.09, \mathrm{p}=0.38)$. The NTISS at the first SAT (15.6, range 1-27) was higher than the NTISS at the second SAT $(12.9$, range $0-25)(p<0.001)$. However, the decrease in the $\mathrm{L} / \mathrm{M}$ ratio was not related to the decrease in the NTISS $(r=-0.07, \mathrm{p}=0.50)$.

In multiple regression analysis of perinatal factors, only umbilical arterial $\mathrm{pH}$ correlated somewhat with the $\mathrm{L} / \mathrm{M}$ ratio measured within two days of birth $(\mathrm{T}=-1.98, \mathrm{p}=0.05)$.

\section{Preterm versus term infants}

Sixteen term infants were tested within two days of birth. In nine of them, a second SAT was performed three to six days 


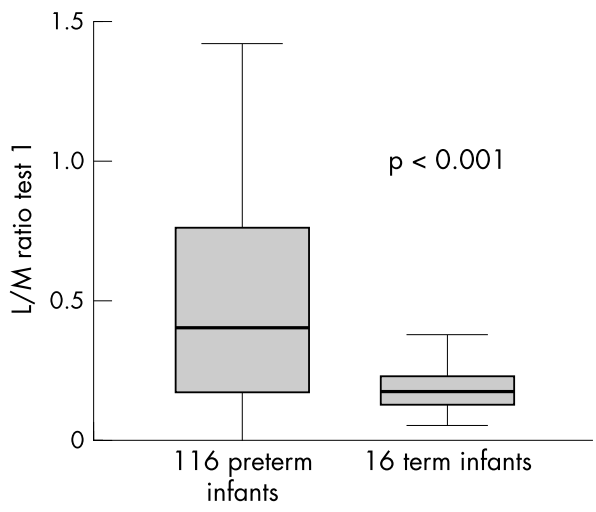

Figure 3 Lactulose/mannitol (L/M) ratio in 116 preterm infants and 16 healthy term infants measured within two days of birth (test 1). It was significantly higher in the preterm than the term infants $(0.404$ and 0.170 respectively, $p<0.001)$.

after the first test. In the remaining seven, the second SAT was not performed because of early discharge or failure to collect urine. The L/M ratio in preterm infants was higher than in term infants in the first test $(0.404$ and 0.170 respectively, $\mathrm{p}<0.005$; fig 3 ) because of a lower permeability to mannitol $(\mathrm{p}=0.05)$. However, in the second SAT, the $\mathrm{L} / \mathrm{M}$ ratio in preterm infants was not different from that in term infants $(0.182$ and 0.123 respectively, $p=0.08)$. Excluding "critically ill" preterm infants from our study did not change the differences found between preterm and term infants.

\section{DISCUSSION}

In our study, the largest study in neonates, intestinal permeability, measured by the sugar absorption test, in preterm infants less than 2 days old was not related to gestational age or birth weight. However, it was higher in preterm infants than in healthy term infants ( $>37$ weeks of gestational age) but only if measured within two days of birth. At a postnatal age of 4-7 days, intestinal permeability was not different in preterm infants and term infants. Our findings are in line with the results of Shulman et $a^{8}$ and Rouwet et al, ${ }^{9}$ who found that intestinal permeability was not different in infants with a gestational age of $<28$ weeks compared with infants with a gestational age of $>28$ weeks. However, in the study of Shulman et $a l^{8}$, intestinal permeability was not measured before the postnatal age of 10 days. In two other studies, however, intestinal permeability in preterm infants was found to be related to gestational age. ${ }^{13}$ Several methodological differences exist between our study and the latter studies. In those studies, the so called steady state method for the measurement of intestinal permeability was used. The osmolarity of the test solution in intestinal permeability tests is known to influence the test results. ${ }^{18}{ }^{19}$ Although Weaver $\mathrm{et} \mathrm{al}^{3}$ state that the osmolarity of the feeds was unchanged by the addition of lactulose and mannitol, in neither that study nor the one of Beach et al was the osmolarity of the test solution reported. We used the single load method as we have used previously in older children and adults. ${ }^{11}$ This method has the advantage that only one (small) dose of the test solution needs to be given. Therefore, the test is also feasible in very sick infants who do not tolerate normal amounts of enteral feeding. Intestinal permeability tests, measuring the ratio of two macromolecules in urine, are less influenced by variables such as gastric emptying and renal excretion. Furthermore, the ratio of two macromolecules in urine depends less on complete urine collection for six hours, which is in accordance with the results of Akram et $a l^{20}$ who found no difference in intestinal permeability between a two hour urine collection and a five to six hour urine collection in adults. The results of our study are in line with these findings, as we did not find any difference between preterm infants in whom urine collection was complete and preterm infants in whom urine collection was incomplete but at least $5 \mathrm{ml}$.

Besides the method of measurement of intestinal permeability, the study design (including postnatal age and gestational age) may influence the test results. In the study of Beach et al, ${ }^{1}$ infants with a gestational age of 26-29 weeks were first studied one to two weeks after birth, whereas the infants with a gestational age of 31-36 weeks were studied three times within the first two weeks. Therefore, it is unclear at which postnatal age the infants were studied. As postnatal factors, including postnatal age, have a large effect on intestinal permeability, as found in our study and others, ${ }^{3689}$ it is difficult to draw any conclusions from the study of Beach et al. ${ }^{1}$ In the study of Weaver et $a l,{ }^{3}$ the results are expressed only as days after the start of oral feeding and not as postnatal age. Therefore, the postnatal age of the infants is unclear. If the preterm infants in our study are separated into two groups ( $<34$ weeks and $>34$ weeks gestation), no differences between the groups were found in intestinal permeability measured within two days of birth or three to six days later. The differences in results for the preterm infants $(<34$ weeks gestation) between the study of Weaver et al in 1983 and ours may also be due to changes in perinatal treatment since that time, for example the use of antenatal steroids. In the study of Shulman et al $^{8}$, antenatal steroid administration was only associated with decreased intestinal permeability at the postnatal age of 28 days; intestinal permeability was not different at a postnatal age of 10 days, which is in line with our results from the second test at the postnatal age of 4-7 days. In our study, intestinal permeability was not different in preterm infants exposed to antenatal steroids $(n=70)$ and preterm infants not exposed to antenatal steroids $(n=46)$, both measured within two days of birth and three to six days later.

Besides differences in postnatal age, severity of illness may influence intestinal permeability. In critically ill adults, intestinal permeability is increased compared with healthy controls. ${ }^{1621} 22$ In contrast with previous studies, we included both "healthy" and "critically ill" preterm infants. However, intestinal permeability was not different in "critically ill" preterm infants (infants on a ventilator, suffering from asphyxia, and/or born small for gestational age) compared with "healthy" preterm infants. Excluding "critically ill" preterm infants from our study did not change the differences found between preterm and term infants. Furthermore, although the NTISS decreased between the first and second test, the decrease was not related to the decrease in intestinal permeability between the first and second test. As mentioned above, differences in perinatal treatment may have affected the results of our study compared with the results of the studies performed in the 1980s.

Several other perinatal factors-for example, the amount and type of enteral feeding-may influence intestinal permeability shortly after birth. At the first test, only 30/116 infants received enteral feeding, whereas 95/102 infants had started enteral feeding at the second test. Although most infants had received enteral feeding for only one or two days at the second test, enteral feeding may account for the rapid decrease in intestinal permeability in preterm infants. In the study of Rouwet et al, ${ }^{9}$ intestinal permeability increased between postnatal day 1 and 7 , and decreased between day 7 and 14. As enteral feeding was started in all infants after day 7, this may explain the temporary increase in intestinal permeability as stated by the authors. ${ }^{9}$ Because many infants in our study were fed a mixture of mother's milk and preterm formula in the first days of life, we were unable to determine the influence of the type of enteral feeding on intestinal permeability. Of the perinatal factors evaluated in our study in preterm infants, only arterial umbilical $\mathrm{pH}$ correlated with intestinal permeability measured within two days of birth. A low arterial umbilical $\mathrm{pH}$ is thought to reflect an impaired 
condition of the fetus shortly before birth. This may influence the condition of the small bowel leading to increased intestinal permeability, which in turn may increase the risk of necrotising enterocolitis. However, to our knowledge, no other studies have looked at arterial umbilical $\mathrm{pH}$ in preterm infants in relation to intestinal permeability or necrotising enterocolitis.

Although in preterm infants, intestinal permeability was not related to gestational age, it was higher in preterm infants than in healthy term infants. We hypothesise that the continuous maturation of the intestinal mucosa may decrease intestinal permeability in the last few weeks of pregnancy. In addition, the causes of preterm birth-for example, maternal infection-may play a role in the increased intestinal permeability in preterm infants shortly after birth compared with healthy term infants who are born after elective caesarean section.

In summary, we have shown that intestinal permeability, measured within two days of birth by a single load sugar absorption test, is not related to gestational age or birth weight in preterm infants 26-37 weeks of gestational age. However, it is higher in preterm infants than in term infants during the first 2 days of life, but not different at 4-7 days of age. Intestinal permeability in preterm infants decreases in the first week of life, suggesting that postnatal factors play an important role in the rapid adaptation of the small intestine to the extrauterine circumstances.

\section{ACKNOWLEDGEMENTS}

We are grateful to R A van Lingen MD PhD and E W A Smink RN for their invaluable support in the recruitment of patients and data collection.

Authors' affiliations

R M van Elburg, W P F Fetter, Department of Neonatology, VU

University Medical Center, Amsterdam, The Netherlands

$\mathbf{R} \mathbf{M}$ van Elburg, W P F Fetter, C M Bunkers, Department of

Neonatology, Isala Clinics, Location Sophia, Zwolle, The Netherlands

H S A Heymans, Department of Pediatrics, Emma Children's Hospital,

Academic Medical Center, Amsterdam

\section{REFERENCES}

1 Beach R, Menzies IS, Clayden GS, et al. Gastrointestinal permeability changes in the preterm neonate. Arch Dis Child 1982;57:141-5.
2 Catassi C Bonucci A, Coppa GV et al. Intestinal permeability changes during the first month: effect of natural versus artificial feeding. J Pediatr Gastroenterol Nutr 1995;21:383-6.

3 Weaver LT, Laker MF, Nelson R. Intestinal permeability in the newborn. Arch Dis Child 1984;59:236-41.

4 Insoft RM, Sanderson IR, Walker WA. Development of immune function in the intestine and its role in neonatal diseases. Pediatr Clin North Am 1996:43:551-71.

5 Veereman-Wauters G. Neonatal gut development and postnata adaptation. Eur J Pediatr 1996;155:627-32.

6 Weaver LT, Laker MF, Nelson R, et al. Milk feeding and changes in intestinal permeability and morphology in the newborn. J Pediatr Gastroenterol Nutr 1987;6:351-8.

7 Weaver LT. Soy-based infant milk formulas and passive intestinal permeability. Lancet 1989;1:1023-4.

8 Shulman RJ, Schanler RJ, Lau C, et al. Early feeding, antenatal glucocorticoids, and human milk decrease intestinal permeability in preterm infants. Pediatr Res 1998:44:519-23.

9 Rouwet EV, Heineman E, Buurman WA, et al. Intestinal permeability and carrier-mediated monosaccharide absorption in preterm neonates during the early postnatal period. Pediatr Res 2002;51:64-70.

10 van Elburg RM, Uil JJ, Mulder CJJ, et al. Intestinal permeability in patients with coeliac disease and relatives of patients with coeliac disease. Gut 1993:34:354-7.

11 van Elburg RM, Uil JJ, Kokke FTM, et al. Repeatability of the sugar absorption test, using lactulose and mannitol, for measuring intestinal permeability for sugars. J Pediatr Gastroenterol Nutr 1995;20:184-8.

12 van Elburg RM, Van Aalderen WMC, Uil JJ, et al. Intestinal permeability in exocrine pancreatic insufficiency due to cystic fibrosis or chronic pancreatitis. Pediatr Res 1996:39:985-91.

13 Uil JJ, Van Elburg RM, van Overbeek FM, et al. Follow-up of treated coeliac disease patients: sugar absorption test and intestinal biopsies compared. Eur J Gastroenterol Hepatol 1996;8:219-23.

14 Uil JJ, Van Elburg RM, van Overbeek FM, et al. Clinical implications of the sugar absorption test: intestinal permeability to assess mucosal barrier function. Scand J Gastroenterol Suppl 1997;32:70-8.

$15 \mathrm{Neu} \mathrm{J}$. Necrotizing enterocolitis. The search for a unifying pathogenic theory leading to prevention. Pediatr Clin North Am 1996;43:409-32

16 Harris CE, Griffiths RD, Freestone N, et al. Intestinal permeability in the critically ill. Intensive Care Med 1992;18:38-41.

17 Gray JE, Richardson DK, McCormick MC, et al. Neonatal therapeutic intervention scoring system: a therapy-based severity-of-illness index. Pediatrics 1992;90:561-7.

18 Peeters $M$, Hiele M, Ghoos $H$, et al. Test conditions greatly influence permeation of water soluble molecules through the intestinal mucosa: need for standardisation. Gut 1994:35:404-8.

19 Uil JJ, van Elburg RM, Janssens PMW, et al. Sensitivity of a hyperosmolar or "low"-osmolar test solution for the sugar absorption in recognizing small intestinal mucosal damage in coeliac disease. Digest Liver Dis 2000;32:195-200.

20 Akram S, Murani S, Ou CN, et al. Assessment of intestinal permeability with a two-hour urine collection. Dig Dis Sci 1998:43:1946-50.

21 Hadfield RJ, Sinclair DG, Houldsworth PE, et al. Effects of enteral and parenteral nutrition on gut mucosal permeability in the critically ill. Am J Respir Crit Care Med 1995;152:1545-8.

22 Roumen RM, Hendriks T, Wevers RA, et al. Intestinal permeability after severe trauma and hemorrhagic shock is increased without relation to septic complications. Arch Surg 1993;128:453-7. 